\title{
La gonorrhée au Canada de 2010 à 2015
}

\author{
Y Choudhri', J Miller ${ }^{1}$, J Sandhu' ${ }^{1}$ A Leon ${ }^{1}$, J Aho ${ }^{1 *}$
}

\section{Résumé}

Mise en contexte : La gonorrhée est la deuxième infection transmissible sexuellement (ITS) la plus fréquemment signalée au Canada après la chlamydia et les taux de cette infection sont en augmentation depuis 1997.

Objectif : Résumer les tendances observées dans les taux de gonorrhée au Canada de 2010 à 2015.

Méthodologie : Les cas de gonorrhée confirmés en laboratoire sont déclarés à l'Agence de la santé publique du Canada (ASPC) par l'ensemble des provinces et des territoires canadiens. Ainsi, le taux d'infection gonococcique a pu être calculé à l'échelle nationale, de même que par sexe, par groupe d'âge et par province ou le territoire.

Résultats : En 2015, 19845 cas de gonorrhée ont été déclarés au Canada, ce qui correspondait à un taux de 55,4 cas par 100000 habitants et à une augmentation de 65,4\% depuis 2010 (33,5 cas par 100000 habitants). Les hommes présentent systématiquement des taux plus élevés que les femmes (70,2 cas par 100000 habitants comparativement à 40,6 cas par 100000 habitants en 2015$)$ et un accroissement plus rapide des taux $(85,2 \%$ comparativement à $39,5 \%$ entre 2010 et 2015). Les taux chez les les adultes de 60 ans et plus ont augmenté plus rapidement que les taux chez les personnes plus jeunes, bien que les taux absolus les plus élevés étaient retrouvés chez les personnes de 15 à 29 ans. Les Territoires du Nord-Ouest, le Nunavut et le Yukon présentaient les taux de gonorrhée les plus élevés en 2015.

Conclusion : Les hommes, les adolescents et les jeunes adultes continuent de représenter la majorité des cas de gonorrhée. Davantage de recherches sont nécessaires afin de mieux comprendre les tendances actuelles de l'épidémiologie de la gonorrhée afin de maintenir, d'évaluer et d'améliorer les interventions de prévention primaire et secondaire des infections transmissibles sexuellement.

\begin{abstract}
Affiliation
${ }^{1}$ Centre de la lutte contre les maladies transmissibles et les infections, Agence de la santé publique du Canada, Ottawa (Ontario)
\end{abstract}

${ }^{\star}$ Correspondance : josephine. aho@canada.ca

Citation proposée : Choudhri Y, Miller J, Sandhu J, Leon A, Aho J. La gonorrhée au Canada de 2010 à 2015. Relevé des maladies transmissibles au Canada. 2018;44(2):40-6. https://doi.org/10.14745/ccdr.v44i02a01f

\section{Introduction}

La gonorrhée, causée par la bactérie Neisseria gonorrhoeae, est la deuxième infection transmissible sexuellement (ITS) la plus fréquemment déclarée au Canada, après la chlamydia. On estime que 78 millions de cas de gonorrhée sont advenus dans le monde en 2012 (1). La plupart des infections sont génitales, mais des infections pharyngées et anorectales peuvent survenir. Les infections gonococciques sont habituellement asymptomatiques chez les femmes, mais symptomatiques chez les hommes (2). Chez les femmes, les symptômes peuvent comprendre des pertes vaginales; chez les hommes, les symptômes consistent souvent en une miction douloureuse, un écoulement urétral anormal et une enflure des testicules (3). Une gonorrhée qui n'est pas traitée peut mener à de l'arthrite réactive, à une infection gonococcique disséminée et à l'infertilité chez les deux sexes (bien qu'elle soit rare chez les hommes) (2). Les conséquences cliniques d'une gonorrhée non traitée comprennent une maladie inflammatoire pelvienne, des douleurs pelviennes chroniques et le risque d'une grossesse ectopique chez la femme; chez I'homme, les conséquences cliniques comprennent une orchiépididymite (2). La transmission de la mère à l'enfant au moment de la naissance peut entraîner une conjonctivite chez les nouveau-nés, avec une possible évolution vers la cécité si l'infection n'est pas détectée et traitée rapidement (4). La gonorrhée accroît aussi le caractère infectieux et la vulnérabilité au VIH en augmentant le nombre de cellules cibles du VIH présentes dans les voies génitales et en amplifiant l'excrétion du $\mathrm{VIH}$ (lorsqu'une cellule infectée libère de particules virales qui peuvent à leur tour infecter de nouvelles cellules) $(5,6)$.

Depuis 1997, le Canada a été témoin d'une augmentation des taux de gonorrhée dans la plupart de ses provinces et de ses territoires, ce qui a augmenté le fardeau de la maladie sur notre système de soins de santé (2). En 2012, le taux global de gonorrhée était de 36,2 par 100000 habitants, une augmentation de 38,9\% comparativement au taux de 2003 (7). Comme par les années antérieures, davantage de cas ont été signalés chez les hommes que chez les femmes (dans un ratio de $1: 1,3)$, mais l'augmentation relative du taux était plus importante chez les femmes (7). De plus, le contrôle et le traitement de la gonorrhée sont devenus beaucoup plus complexes en raison du développement de la résistance antimicrobienne dans plusieurs pays, dont le Canada (8). L'émergence récente de souches 
résistantes à l'azithromycine et la diminution de la sensibilité aux céphalosporines menacent les dernières options de traitement disponibles (9). En 2014, 52,4 \% des souches de N. gonorrhoeae isolées au Canada étaient résistantes à au moins l'un des antibiotiques testés.

L'objectif du présent article est de résumer les tendances observées dans les taux d'infections à la gonorrhée déclarées au Canada de 2010 à 2015.

\section{Méthodologie}

\section{Sources des données}

La gonorrhée est une maladie à déclaration obligatoire au pays depuis 1924. Les autorités provinciales et territoriales de la santé fournissent des données non nominatives portant sur les cas confirmés en laboratoire à l'Agence de la santé publique du Canada (ASPC) au moyen du Système canadien de surveillance des maladies à déclaration obligatoire (SCSMDO) (10). Les définitions des cas confirmés sont présentées en annexe (11).

Les variables fournies avec le diagnostic comprennent l'âge au moment du diagnostic, l'année du diagnostic, la province ou le territoire où le diagnostic a été posé et le sexe. Les données reçues sont validées en collaboration avec la province ou le territoire en question. Les données du $1^{\text {er }}$ janvier 2010 au 31 décembre 2015 ont été obtenues de toutes les provinces et de tous les territoires; elles ont été extraites du SCSMDO en juillet 2017.

\section{Analyse des données}

Une analyse descriptive a été réalisée à l'aide de Microsoft Excel. Les taux nationaux annuels des cas signalés ont été calculés en utilisant le nombre de cas extrait du SCSMDO comme numérateur et l'estimation de la taille de la population annuelle fournies par Statistiques Canada comme dénominateurs. Les taux spécifiques au sexe, à l'âge, à la province ou au territoire ont aussi été calculés. Pour l'ensemble des années, les taux sont présentés pour 100000 habitants. Aucune procédure statistique n'a été employée pour les analyses comparatives. Les petits nombres sont plus sensibles aux variations et, de ce fait, les taux correspondants devraient être interprétés avec prudence. Les rapports précédents peuvent présenter des taux différents pour certaines années en raison des retards de déclaration et de la mise à jour des données.

\section{Résultats}

Entre 2010 et 2015, le nombre de cas de gonorrhée signalés a augmenté, passant de 11386 à 19845 cas. Le taux global associé était de 55,4 cas par 100000 habitants en 2015 (par rapport à 33,5 cas par 100000 habitants en 2010) (figure 1).

\section{Sexe et âge}

Au cours de la période de surveillance 2010-2015, le taux de gonorrhée était plus élevé chez les hommes que chez les femmes (70,2 cas par 100000 hommes comparativement à 40,6 cas par 100000 femmes en 2015). De plus, le taux des hommes a augmenté à un rythme plus soutenu que celui des femmes : 85,2 \% (de 37,9 à 70,2 cas par 100000 habitants) comparativement à 39,5\% (de 29,1 à 40,6 par 100000 habitants) (figure 1).

\section{Figure 1 : Taux globaux ${ }^{a}$ et selon le sexe des cas de gonorrhée confirmés en laboratoire et déclarés, de 2010 à 2015, au Canada}

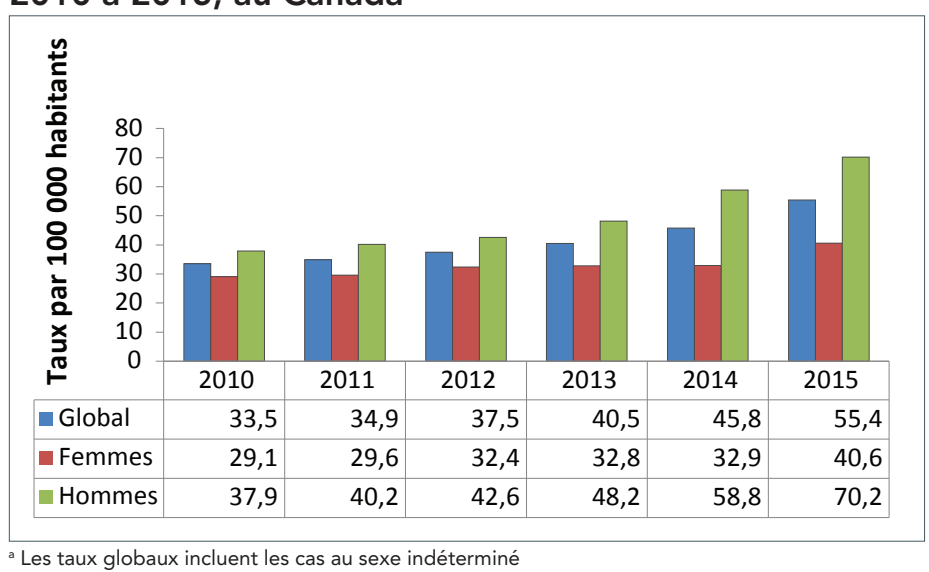

Les adolescents et les jeunes adultes avaient les taux de gonorrhée parmi les plus élevés en 2015 (205,3, 176,7 et 113,4 cas par 100000 habitants pour les personnes ayant respectivement de 20 à 24 ans, de 25 à 29 ans et de 15 à 19 ans). Les taux les moins élevés étaient retrouvés chez les enfants de moins de 10 ans (0,6 cas par 100000 habitants) et les personnes de 60 ans et plus (3,3 cas par 100000 habitants).

De 2010 à 2015, les adolescents de 10 à 14 ans formaient le seul groupe d'âge affichant une diminution des taux de gonorrhée (-13,5\%, soit de 4,6 à 4 cas par 100000 habitants). À l'opposé, les adultes de 30 à 39 ans présentaient la plus forte augmentation de taux $(128,7 \%$, soit de 42,9 à 98,1 cas par 100000 habitants), suivies des personnes âgées de 40 à 59 ans (100\%, soit de 14,1 à 28,2 cas par 100000 habitants).

Lorsque les taux par groupes d'âge étaient stratifiés selon le sexe, les taux des femmes de moins de 20 ans étaient systématiquement plus élevés que ceux des hommes du même âge (ces données ne sont pas présentées). De manière générale, les taux des hommes augmentaient plus rapidement pour tous les groupes d'âge, à l'exception du groupe des jeunes de 10 à 14 ans, chez lequel une diminution relative de $55 \%$ a été observée chez les garçons (et une diminution de $1 \%$ chez les filles).

\section{Distribution géographique}

Les taux et le nombre de cas pour chaque province et chaque territoire sont présentés au tableau 1. Seuls le Nunavut et le Nouveau-Brunswick ont eu une diminution du nombre de cas signalés de gonorrhée (des diminutions respectives de $56,9 \%$ et $22 \%$ ). Le Nunavut, de même que les Territoires du Nord-Ouest et le Yukon, avaient les taux les plus élevés en 2015. Les provinces de l'Atlantique (la Nouvelle-Écosse, le Nouveau-Brunswick, l'île-du-Prince-Édouard et Terre-Neuve-et-Labrador) présentaient les taux rapportés les plus bas. Les provinces et les territoires présentant l'augmentation 
de taux la plus élevée au cours de la période étaient le Yukon (237,3 \%), Terre-Neuve-et-Labrador (212,7\%), I'Alberta (159,8 \%) et la Colombie-Britannique $(143,7 \%)$.

\section{Tableau 1 : Nombre de cas et taux de gonorrhée confirmés en laboratoire et déclarés, par province et par territoire au Canada, de 2010 à 2015}

\begin{tabular}{|c|c|c|c|c|c|c|}
\hline \multirow{2}{*}{$\begin{array}{l}\text { Province } \\
\quad \text { ou } \\
\text { territoire }\end{array}$} & \multicolumn{6}{|c|}{$\begin{array}{l}\text { Cas confirmés en laboratoire selon l'année du } \\
\text { diagnostic (taux par } 100000 \text { habitants) }\end{array}$} \\
\hline & 2010 & 2011 & 2012 & 2013 & 2014 & 2015 \\
\hline Alberta & $\begin{array}{l}1182 \\
(31,7)\end{array}$ & $\begin{array}{l}1508 \\
(39,8)\end{array}$ & $\begin{array}{l}2103 \\
(54,2)\end{array}$ & $\begin{array}{l}2017 \\
(50,5)\end{array}$ & $\begin{array}{l}1908 \\
(46,4)\end{array}$ & $\begin{array}{l}3438 \\
(82,3)\end{array}$ \\
\hline $\begin{array}{l}\text { Colombie- } \\
\text { Britannique }\end{array}$ & $\begin{array}{l}1365 \\
(30,6)\end{array}$ & $\begin{array}{l}1649 \\
(36,7)\end{array}$ & $\begin{array}{l}1420 \\
(31,2)\end{array}$ & $\begin{array}{l}1841 \\
(40,1)\end{array}$ & $\begin{array}{l}2031 \\
(43,7)\end{array}$ & $\begin{array}{l}3495 \\
(74,5)\end{array}$ \\
\hline Manitoba & $\begin{array}{r}982 \\
(80,4)\end{array}$ & $\begin{array}{l}1055 \\
(85,5)\end{array}$ & $\begin{array}{r}1349 \\
(107,9)\end{array}$ & $\begin{array}{l}1217 \\
(96,2)\end{array}$ & $\begin{array}{l}1107 \\
(86,4)\end{array}$ & $\begin{array}{l}1085 \\
(83,7)\end{array}$ \\
\hline $\begin{array}{l}\text { Nouve } \\
\text { Brunsv }\end{array}$ & $\begin{array}{r}64 \\
(8,5)\end{array}$ & $\begin{array}{r}64 \\
(8,5)\end{array}$ & $\begin{array}{r}38 \\
(5,0)\end{array}$ & $\begin{array}{r}47 \\
(6,2)\end{array}$ & $\begin{array}{r}44 \\
(5,8)\end{array}$ & $\begin{array}{r}50 \\
(6,6)\end{array}$ \\
\hline $\begin{array}{l}\text { Terre- } \\
\text { et-Lak }\end{array}$ & $\begin{array}{r}12 \\
(2,3)\end{array}$ & $\begin{array}{r}26 \\
(5,0)\end{array}$ & $\begin{array}{r}16 \\
(3,0)\end{array}$ & $\begin{array}{r}41 \\
(7,8)\end{array}$ & $\begin{array}{r}66 \\
(12,5)\end{array}$ & $\begin{array}{r}38 \\
(7,2)\end{array}$ \\
\hline $\begin{array}{l}\text { Territoires du } \\
\text { Nord-Ouest }\end{array}$ & $\begin{array}{r}219 \\
(506,0)\end{array}$ & $\begin{array}{r}143 \\
(328,7)\end{array}$ & $\begin{array}{r}192 \\
(440,4)\end{array}$ & $\begin{array}{r}97 \\
(221,5)\end{array}$ & $\begin{array}{r}245 \\
(558,2)\end{array}$ & $\begin{array}{r}361 \\
(815,9)\end{array}$ \\
\hline $\begin{array}{l}\text { Nouv } \\
\text { Écoss }\end{array}$ & $\begin{array}{r}100 \\
(10,6)\end{array}$ & $\begin{array}{r}102 \\
(10,8) \\
\end{array}$ & $\begin{array}{r}119 \\
(12,6) \\
\end{array}$ & $\begin{array}{r}97 \\
(10,3) \\
\end{array}$ & $\begin{array}{r}114 \\
(12,1)\end{array}$ & $\begin{array}{r}133 \\
(14,1) \\
\end{array}$ \\
\hline Nunavut & $\begin{array}{r}648 \\
(1942,9)\end{array}$ & $\begin{array}{r}595 \\
(1740,0)\end{array}$ & $\begin{array}{r}448 \\
(1290,8)\end{array}$ & $\begin{array}{r}466 \\
(1316,3)\end{array}$ & $\begin{array}{r}326 \\
(905,0)\end{array}$ & $\begin{array}{r}306 \\
(837,6)\end{array}$ \\
\hline Ontario & $\begin{array}{l}3966 \\
(30,2)\end{array}$ & $\begin{array}{l}4205 \\
(31,7)\end{array}$ & $\begin{array}{l}4097 \\
(30,5)\end{array}$ & $\begin{array}{l}4540 \\
(33,5)\end{array}$ & $\begin{array}{l}5840 \\
(42,7)\end{array}$ & $\begin{array}{l}5932 \\
(43,0)\end{array}$ \\
\hline $\begin{array}{l}\text { Île-du-Prince- } \\
\text { Édouard }\end{array}$ & $\begin{array}{r}0 \\
(0,0)\end{array}$ & $\begin{array}{r}11 \\
(7,6) \\
\end{array}$ & $\begin{array}{r}8 \\
(5,5) \\
\end{array}$ & $\begin{array}{r}6 \\
(4,1) \\
\end{array}$ & $\begin{array}{r}7 \\
(4,8)\end{array}$ & $\begin{array}{r}10 \\
(6,8)\end{array}$ \\
\hline Québ & $\begin{array}{l}2054 \\
(25,9)\end{array}$ & $\begin{array}{l}1864 \\
(23,3)\end{array}$ & $\begin{array}{l}2219 \\
(27,4)\end{array}$ & $\begin{array}{l}2642 \\
(32,4)\end{array}$ & $\begin{array}{l}3312 \\
(40,3)\end{array}$ & $\begin{array}{l}3927 \\
(47,5)\end{array}$ \\
\hline Saska & $\begin{array}{r}763 \\
(72,6) \\
\end{array}$ & $\begin{array}{r}758 \\
(71,1)\end{array}$ & $\begin{array}{l}1018 \\
(93,7)\end{array}$ & $\begin{array}{r}1213 \\
(109,8) \\
\end{array}$ & $\begin{array}{r}1240 \\
(110,6)\end{array}$ & $\begin{array}{r}957 \\
(84,5) \\
\end{array}$ \\
\hline Yukon & $\begin{array}{r}31 \\
(89,6)\end{array}$ & $\begin{array}{r}6 \\
(16,9)\end{array}$ & $\begin{array}{r}9 \\
(25,0)\end{array}$ & $\begin{array}{r}10 \\
(27,5) \\
\end{array}$ & $\begin{array}{r}49 \\
(132,9)\end{array}$ & $\begin{array}{r}113 \\
(302,2)\end{array}$ \\
\hline Canada & $\begin{array}{r}11386 \\
(33,5)\end{array}$ & $\begin{array}{r}11986 \\
(34,9)\end{array}$ & $\begin{array}{r}13036 \\
(37,5)\end{array}$ & $\begin{array}{r}14234 \\
(40,5)\end{array}$ & $\begin{array}{r}16289 \\
(45,8)\end{array}$ & $\begin{array}{r}19845 \\
(55,4)\end{array}$ \\
\hline
\end{tabular}

\section{Discussion}

Après une diminution marquée entre le début des années 1980 et les années 1990, les taux de gonorrhée se sont accrus à la fin des années 1990 au Canada et ils ont poursuivi leur croissance de 2010 à 2015 parmi les hommes et les femmes de presque tous les groupes d'âge. D'autres pays ont présenté des tendances similaires. Par exemple, les États-Unis ont vu leur taux augmenter de $22,2 \%$ chez les hommes et de $13,8 \%$ chez les femmes de 2015 à 2016 (12,13). En accord avec la tendance observée entre 2012 et 2014, le Canada présentait le taux de cas déclarés de gonorrhée le moins élevé en 2015 comparativement aux États-Unis, à l'Australie et à l'Angleterre (55,4 cas par 100000 habitants comparativement à $123,9,79,7$ et 75,3 cas par 100000 habitants réspectivement) (13-15).
Plusieurs facteurs pourraient expliquer l'augmentation apparente des taux de gonorrhée. L'introduction d'un outil diagnostique plus sensible, les tests d'amplification des acides nucléiques (TAAN), a augmenté de manière significative le nombre de cas détectés (16). De plus, les directives nationales en matière de traitement, au Canada comme ailleurs, ont mis l'accent sur l'importance de procéder à des tests de dépistage sur d'autres sites anatomiques (infections oropharyngées et rectales) chez certaines populations, ce qui pourrait avoir augmenté le nombre de cas détectés (2).

Combinée à l'amélioration du dépistage et de la détection, la résistance antimicrobienne aux antibiotiques de première ligne contribue aussi aux taux élevés de gonorrhée (17). Les infections gonococciques ont récemment démontré une résistance ou une diminution de leur sensibilité à tous les antibiotiques couramment employés en traitement au Canada, y compris la pénicilline, les tétracyclines, les macrolides et les quinolones $(8,18)$. La résistance antimicrobienne peut entraîner une inefficacité du traitement et mener à une transmission continue de l'infection non traitée. Une étude réalisée aux États-Unis a observé une résistance aux antibiotiques plus élevée chez les hommes ayant des relations sexuelles avec d'autres hommes (HARSAH). Ce phénomèene peut également avoir contribué à l'augmentation des taux chez les Canadiens de sexe masculin (19). Les directives canadiennes en matière de traitement de la gonorrhée ont fréquemment été mises à jour au cours des cinq dernières années afin de refléter les nouvelles informations concernant la résistance antimicrobienne de $N$. gonorrhoeae. Cependant, une étude récente réalisée en Ontario a démontré une faible adhésion aux directives de traitement de la gonorrhée (20). Un traitement inefficace affecte le patient et peut augmenter la transmission de souches résistantes (17). Par ailleurs, la surveillance des souches résistantes s'est avérée plus difficile en raison de l'utilisation croissante des TAAN pour le dépistage. Or, cet outil diagnostique ne permet pas l'évaluation de la sensibilité aux antimicrobiens. Cependant, de nouvelles méthodes moléculaires de détection utilisant des réactions de polymérisation en chaîne, le polymorphisme mononucléotidique ou le séquençage dans le but d'évaluer la résistance sont explorées actuellement afin d'améliorer le dépistage de souches résistantes de la gonorrhée $(21,22)$.

Contrairement à la chlamydia, la plupart des cas de gonorrhée au Canada ont été signalés chez les hommes, comme aux États-Unis, en Australie et en Angleterre (13-15). Cependant, les taux de gonorrhée au Canada étaient supérieurs chez les femmes de moins de 20 ans que chez les hommes du même âge.

L'augmentation des taux de gonorrhée chez les hommes peut s'expliquer en partie par la croissance rapide des taux de gonorrhée dans la population des HARSAH $(23,24)$. Des observations tirées de la littérature démontrent que certains HARSAH ont apporté des changements à leur comportement sexuel, telle la séroadaptation, comme stratégie de réduction des effets nocifs de l'infection au VIH (25). La séroadaptation comprend le sérotriage, défini comme le choix d'avoir des relations sexuelles non protégées avec un partenaire sexuel dont on sait qu'il partage le même statut sérologique, dans le but de réduire le risque de contracter ou de transmettre le $\mathrm{VIH}$ (25). Cependant, ces comportements peuvent augmenter le risque 
de contracter d'autres ITS $(25,26)$. Un autre facteur qui peut expliquer l'augmentation des taux chez les hommes est que la gonorrhée a tendance à être davantage symptomatique chez les hommes que chez les femmes, ce qui peut motiver les hommes à rechercher plus fréquemment un soutien médical et, par conséquent, à obtenir un diagnostic (23). Les taux élevés chez les femmes de moins de 20 ans sont particulièrement inquiétants, étant donné que l'infertilité est une conséquence potentielle de la gonorrhée, ce qui peut entraîner des coûts psychosociaux et économiques substantiels.

Les taux de gonorrhée semblent augmenter à un rythme plus rapide chez les cohortes plus âgées que chez les cohortes plus jeunes. Ce phénomène peut s'expliquer par le fait que la vieillesse s'accompagne de changements physiologiques (sécheresse vaginale), psychosociaux (perte d'un partenaire) et comportementaux (augmentation des comportements à risques en raison de la disparition de la crainte d'une grossesse) naturels, qui peuvent rendre les adultes plus âgés davantage susceptibles de contracter des ITS $(27,28)$.

\section{Points forts et limites}

Le présent rapport de surveillance présente des données nationales sur la gonorrhée à partir de données collectées par toutes les provinces et tous les territoires. De plus, il décrit les taux sur une période de six ans.

Certaines limites des données devraient être notées. Les données exposées dans le présent rapport sous-estiment probablement le taux d'incidence de la gonorrhée, car certaines infections sont asymptomatiques, non dépistées, non diagnostiquées ou non rapportées. Les pratiques de dépistage, de tests en laboratoire et de déclaration sont différentes d'une province et d'un territoire à l'autre. De ce fait, les comparaisons directes entre les provinces et territoires devraient être effectuées avec prudence. Les obstacles communs à la déclaration de cas comprennent le manque de connaissances sur les maladies à déclarer, le temps requis pour effectuer la déclaration et la perception de la gravité de la maladie $(29,30)$. Cependant, comme les études exhaustives de prévalence et d'incidence sont rares, les taux de cas déclarés fournissent des informations précieuses sur les tendances de la maladie et sur les taux d'incidence minimaux.

Les réinfections sont fréquentes avec la gonorrhée et plus d'une infection peut s'être produite et avoir été signalée pour une personne donnée. Ainsi, le nombre de personnes infectées peut être inférieur au nombre de cas signalés. De plus, les données sur les facteurs de risques ne sont pas disponibles dans le SCSMDO, ce qui limite notre capacité à évaluer les facteurs associés aux taux plus élevés de gonorrhée.

L'absence d'analyse statistique dans le présent rapport de surveillance constitue une limite. Puisque les taux n'ont pas été normalisés en fonction de l'âge, les changements observés dans les taux au cours des trente dernières années pourraient être causés partiellement par des changements dans la structure d'âge des provinces et des territoires de même qu'au Canada de manière générale.

\section{Conclusion}

Au Canada, les taux de gonorrhée ont augmenté de 65,4\% entre 2010 et 2015 . Les hommes continuent de présenter des taux plus élevés que les femmes. Des études indiquent que des tests plus sensibles et une résistance antimicrobienne pourraient expliquer une partie de l'augmentation des cas. La surveillance continue des taux de gonorrhée et de la résistance antimicrobienne aidera à ajuster les recommandations de traitement actuelles. Des données portant sur les facteurs de risques seraient utiles afin d'améliorer la surveillance. Des données de recherche et de surveillance sont nécessaires afin de mieux comprendre l'épidémie de gonorrhée actuelle dans le but de maintenir, d'évaluer et d'améliorer les interventions de prévention primaire et secondaire des ITS, y compris des campagnes de sensibilisation aux pratiques sexuelles plus sûres, le dépistage, la recherche de cas et la notification au partenaire.

\section{Déclaration des auteurs}

Y.C. - Conceptualisation, méthodologie, rédaction - première ébauche

J.M. - Logiciel, collecte et conservation des données, validation, analyse formelle, visualisation, rédaction - examen et révision J.S. - Rédaction - première ébauche, visualisation

A.L. - Validation, analyse formelle, visualisation, rédaction examen et révision

J.A. - Conceptualisation, rédaction - première ébauche

\section{Conflit d'intérêt}

Aucun.

\section{Contributions}

Chris Archibald : Supervision, rédaction - examen et révision, ressources, administration du projet

Jennifer Siushansian : rédaction - examen et révision

\section{Remerciements}

Il n'aurait pas été possible de publier le présent rapport sans la contribution continuelle et grandement appréciée de toutes les provinces et tous les territoires à la surveillance nationale des infections transmissibles sexuellement.

\section{Financement}

Le présent travail a été soutenu par l'Agence de la santé publique du Canada.

\section{Références}

1. Newman L, Rowley J, Vander Hoorn S, Wijesooriya NS, Unemo M, Low $\mathrm{N}$ et al. Global estimates of the prevalence and incidence of four curable sexually transmitted infections in 2012 based on systematic review and global reporting. 


\section{SURVEILLANCE}

PLoS One 2015 Dec;10(12):e0143304. DOI (http://dx.doi. org/10.1371/journal.pone.0143304). PubMed (https://www. ncbi.nlm.nih.gov/entrez/query.fcgi? cmd=Retrieve\&db=PubMe d\&list_uids=26646541\&dopt=Abstract)

2. Groupe de travail d'experts pour les Lignes directrices canadiennes sur les infections transmissibles sexuellement - . Infections gonococciques. In: Wong T, Latham-Carmanico C, rédacteurs/rédactrice. Prise en charge et traitement d'infections spécifiques, 2013. Ottawa: Agence de la santé publique du Canada; 2013. https://www.canada.ca/fr/ sante-publique/services/maladies-infectieuses/sante-sexuell e-infections-transmissibles-sexuellement/lignes-directricescanadiennes/infections-transmissibles-sexuellement/ lignes-directrices-canadiennes-infections-transmiss ibles-sexuellement-34.html

3. Centers for Disease Control and Prevention. Gonorrhea - CDC fact sheet (detailed version). Atlanta (GA): CDC; 2016 Oct. https://www.cdc.gov/std/gonorrhea/stdfact-gonorrheadetailed.htm

4. Heymann DL, editor. Control of communicable diseases manual. 20th ed. Washington: American Public Health Association; 2015. 729 p.

5. Galvin SR, Cohen MS. The role of sexually transmitted diseases in HIV transmission. Nat Rev Microbiol 2004 Jan;2(1):33-42. DOI (http://dx.doi.org/10.1038/nrmicro794). PubMed (https:// www.ncbi.nlm.nih.gov/entrez/query.fcgi? $\mathrm{cmd}=$ Retrieve\&db=Pu bMed\&list_uids=15035007\&dopt=Abstract).

6. Rebbapragada A, Kaul R. More than their sum in your parts: the mechanisms that underpin the mutually advantageous relationship between HIV and sexually transmitted infections. Drug Discov Today Dis Mech 2007;4(4):237-46. DOI (http:// dx.doi.org/10.1016/j.ddmec.2007.12.003).

7. Totten $S$, MacLean R, Payne E, Severini A. L'infection à Chlamydia trachomatis et le lyphogranulome vénérien au Canada : 2003-2012. Relevé des maladies transmissibles au Canada. 2015;41(2):22-9. http://www.phac-aspc. gc.ca/publicat/ccdr-rmtc/15vol41/dr-rm41-02/assets/ pdf/15vol41_02-fra.pdf

8. Martin I, Sawatzky P, Liu G, Mulvey MR. La résistance aux antimicrobiens de Neisseria gonorrhoeae au Canada : 2009-2013. Relevé des maladies transmissibles au Canada. 2015;41(2):39-44. https://www.canada.ca/fr/sante-publique/ services/rapports-publications/releve-maladie s-transmissibles-canada-rmtc/numero-mensuel/2015-41/ rmtc-volume-41-02-5-fevrier-2015/rmtc-volume-41-02-5fevrier-2015-3.html

9. Agence de la santé publique du Canada. Système de surveillance de la résistance aux antimicrobiens - Rapport de 2016. Ottawa (ON): ASPC; 2016 Sep. https://www.canada.ca/ content/dam/phac-aspc/documents/services/publications/ drugs-health-products/antibiotic-resistance-antibiotique/ antibiotic-resistance-antibiotique-2016-fra.pdf

10. Agence de la santé publique du Canada. Système de surveillance des maladies à déclaration obligatoire rapport canadien : 2005-2008. Ottawa (ON): ASPC; 2012. http:// publications.gc.ca/collections/collection_2012/aspc-phac/ HP40-75-2012-fra.pdf

11. Agence de la santé publique du Canada. Définitions nosologiques des maladies transmissibles faisant l'objet d'une surveillance nationale. Relevé des maladies transmissibles au Canada. 2009;35(S2):S1-134. https://www.canada.ca/ content/dam/phac-aspc/migration/phac-aspc/publicat/ ccdr-rmtc/09pdf/35s2-fra.pdf

12. Centers for Disease Control and Prevention. Sexually transmitted disease surveillance 2016. Atlanta (GA): US Department of Health and Human Services; 2017. https://www.cdc.gov/std/stats16/CDC_2016_STDS_ Report-for508WebSep21_2017_1644.pdf

13. Centers for Disease Control and Prevention. Sexually transmitted disease surveillance 2015. Atlanta (GA): US Department of Health and Human Services; 2016. https:// www.cdc.gov/std/stats15/STD-Surveillance-2015-print.pdf

14. The Kirby Institute. HIV, viral hepatitis and sexually transmissible infections in Australia: annual surveillance report 2016. Sydney (AU): University of New South Wales; 2016. https://kirby.unsw.edu.au/sites/default/files/kirby/report/ SERP_Annual-Surveillance-Report-2016_UPD170627.pdf

15. Public Health England. Table 2: New diagnoses \& rates by gender, sexual risk \& age group, 2012-2016. London (UK): Public Health England. https://www.gov.uk/government/ uploads/system/uploads/attachment_data/file/626361/2016_ Table_2_Selected_STI_diagnoses__rates_by_gender_ sexual_risk__age_group.pdf

16. Cornelisse VJ, Chow EP, Huffam S, Fairley CK, Bissessor M, De Petra $V$ et al. Increased detection of pharyngeal and rectal gonorrhea in men who have sex with men after transition from culture to nucleic acid amplification testing. Sex Transm Dis 2017 Feb;44(2):114-7. DOI (http://dx.doi.org/10.1097/ OLQ.0000000000000553). PubMed (https://www.ncbi.nlm. nih.gov/entrez/query.fcgi?cmd=Retrieve\&db=PubMed\&lis t_uids=27984552\&dopt=Abstract).

17. Singh $A E$, Gratrix J, Read R, Lovgren M, Drews SJ, Romanowski $B$ et al. Neisseria gonorrhoeae multiantigen sequence typing is beneficial in further characterizing gonococcal populations in Alberta, Canada. Sex Transm Dis 2013 Sep;40(9):744-50. DOI (http://dx.doi.org/10.1097/01. olq.0000431356.80840.d4) PubMed (https://www.ncbi.nlm. nih.gov/entrez/query.fcgi?cmd=Retrieve\&db=PubMed\&lis t_uids=23945428\&dopt=Abstract).

18. Agence de la santé publique du Canada. Traitement des infections gonococciques par suite du retrait du marché de la spectinomycine: Déclaration d'orientation pour les traitements de remplacement. Ottawa (ON): ASPC; 2017. https://www.canada.ca/fr/sante-publique/services/ publications/maladies-et-affections/gonorrhee-traitemen t-de-remplacement.html

19. Kirkcaldy RD, Zaidi A, Hook EW 3rd, Holmes KK, Soge O, del Rio $C$ et al. Neisseria gonorrhoeae antimicrobial resistance among men who have sex with men and men who have sex exclusively with women: the Gonococcal Isolate Surveillance Project, 2005-2010. Ann Intern Med 2013 Mar;158(5 Pt 1):3218. DOI (http://dx.doi.org/10.7326/0003-4819-158-5201303050-00004). PubMed (http://dx.doi.org/10.1097/01. olq.0000431356.80840.d4).

20. Dickson C, Taljaard M, Friedman DS, Metz G, Wong T, Grimshaw JM. The antibiotic management of gonorrhoea in Ontario, Canada following multiple changes in guidelines: an interrupted time-series analysis. Sex Transm Infect 2017 Dec;93(8):561-5. DOI (http://dx.doi.org/10.1136/ sextrans-2017-053224). PubMed (https://www.ncbi.nlm. 
26. Mayer KH. Sexually transmitted diseases in men who have sex with men. Clin Infect Dis 2011 Dec;53 Suppl 3:S79-83. DOI (http://dx.doi.org/10.1093/cid/cir696). PubMed (https://www. ncbi.nlm.nih.gov/entrez/query.fcgi?cmd=Retrieve\&db=PubMe d\&list_uids=22080272\&dopt=Abstract).

27. Johnson BK. Sexually transmitted infections and older adults. J Gerontol Nurs 2013 Nov;39(11):53-60. DOl (http://dx.doi. org/10.3928/00989134-20130918-01). PubMed (https://www. ncbi.nlm.nih.gov/entrez/query.fcgi?cmd=Retrieve\&db=PubMe d\&list_uids=24066789\&dopt=Abstract).

28. Poynten IM, Grulich AE, Templeton DJ. Sexually transmitted infections in older populations. Curr Opin Infect Dis 2013 Feb;26(1):80-5. DOI (http://dx.doi.org/10.1097/ QCO.0b013e32835c2173). PubMed (https://www.ncbi.nlm. nih.gov/entrez/query.fcgi? $\mathrm{cmd}=$ Retrieve $\& \mathrm{db}=$ PubMed\&lis t_uids=23221769\&dopt=Abstract).

29. Friedman SM, Sommersall LA, Gardam M, Arenovich T. Déclaration sous-optimale des maladies à déclaration obligatoire dans les services des urgences au Canada : Enquête sur les connaissances, les pratiques et les obstacles perçus chez les médecins d'urgence. Relevé des maladies transmissibles au Canada. 2006 Sep;32(17):187-98. https:// www.canada.ca/content/dam/phac-aspc/migration/ phac-aspc/publicat/ccdr-rmtc/06pdf/cdr3217.pdf

30. Doyle TJ, Glynn MK, Groseclose SL. Completeness of notifiable infectious disease reporting in the United States: an analytical literature review. Am J Epidemiol 2002 May;155(9):866-74. DOI (http://dx.doi.org/10.1093/ aje/155.9.866). PubMed (https://www.ncbi.nlm.nih.gov/ entrez/query.fcgi?cmd=Retrieve\&db=PubMed\&list_ uids $=11978592 \&$ dopt $=$ Abstract $)$. 
Annexe : définitions des cas confirmés de gonorrhée

Données de laboratoire d'infections acquises de manière génitale, extragénitale ou périnatale :

- détection de Neisseria gonorrhoeae par culture

$\mathrm{OU}$

- détection des acides nucléiques de Neisseria gonorrhoeae
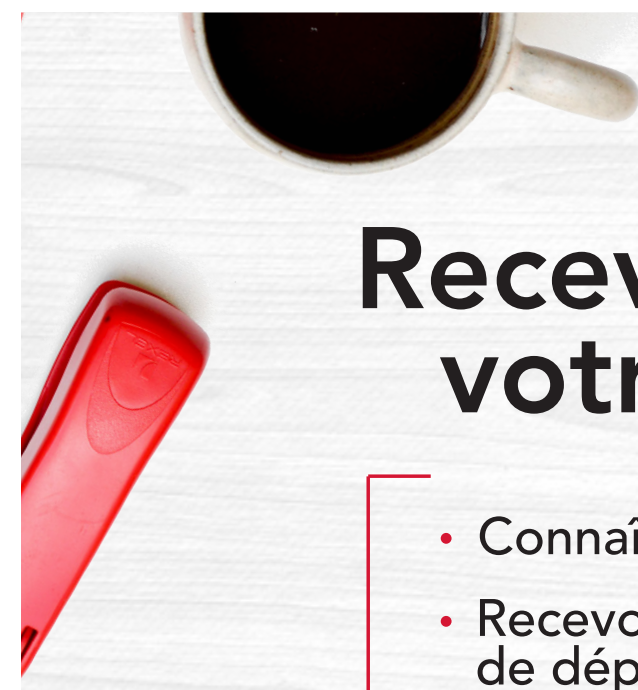

\section{Recevez le RMTC dans votre boîte courriel}

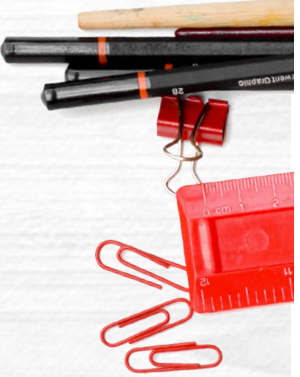

- Connaître les tendances

- Recevoir les directives en matière de dépistage

- Être à l'affût des nouveaux vaccins

- Apprendre sur les infections émergentes

- Recevoir la table des matières directement dans votre boîte courriel
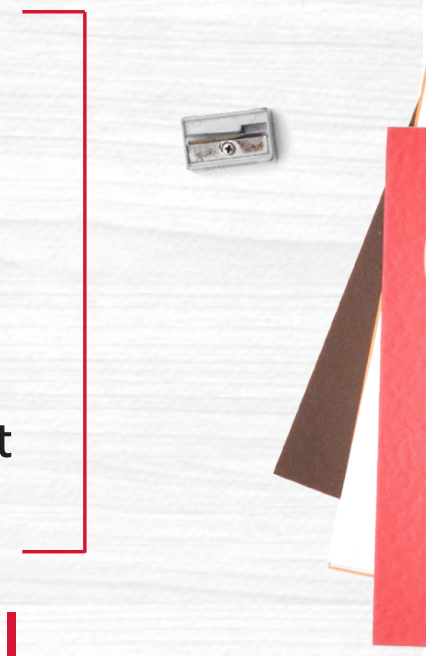

\section{ABONNEZ-VOUS AUJOURD'HUI}

\section{Recherche web : RMTC+abonnez-vous}

Q
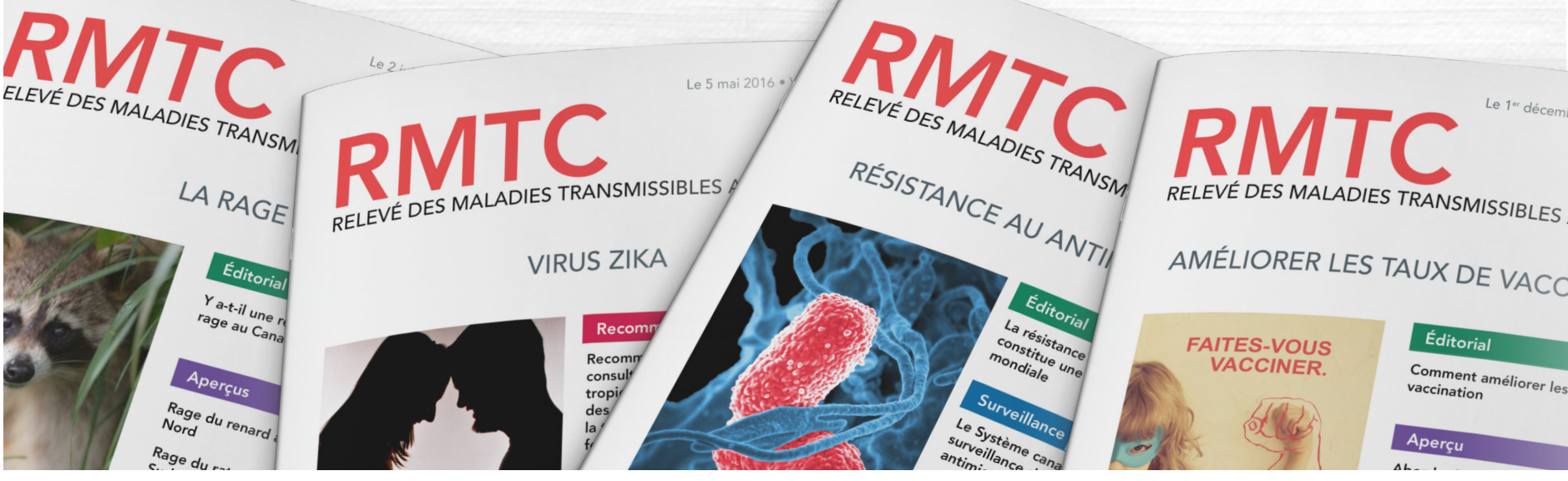

AMÉLIORER LES TAUX DE VACC

FAITES-VOUS

VACCINER.

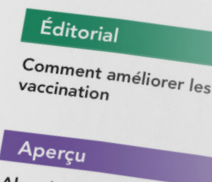

